\title{
In vitro-induction of metronidazole-resistant Giardia duodenalis is not associated with nucleotide alterations in the genes involved in pro-drug activation
}

\author{
Luiz Antonio Pimentel Lopes-Oliveira', Maria Fantinatti', Alda Maria Da-Cruz,2/+ \\ ${ }^{1}$ Fundação Oswaldo Cruz-Fiocruz, Instituto Oswaldo Cruz, Laboratório Interdisciplinar de Pesquisas Médicas, Rio de Janeiro, RJ, Brasil \\ ${ }^{2}$ Universidade do Estado do Rio de Janeiro, Faculdade de Ciências Médicas, Disciplina de Parasitologia, Rio de Janeiro, RJ, Brasil
}

Giardiasis is an infectious disease caused by Giardia duodenalis. The pro-drug metronidazole (MTZ) is the first-line treatment for giardiasis. Parasite's proteins as pyruvate:ferredoxin oxidoreductase (PFOR), ferredoxin (Fd), nitroreductase-1 (NR-1) and thioredoxin reductase (TrxR) participate in MTZ activation. Here, we showed Giardia trophozoites long-term exposed to MTZ presented higher $\mathrm{IC}_{50}$ than controls, showing the drug influenced the parasite survival. That reduction in MTZ's susceptibility does not seem to be related to mutations in the genes $p f o r, f d, n r-1$ or trxr. It points that different mechanism as alterations in other metabolic pathways can account for Giardia resistance to MTZ therapy.

Key words: Giardia duodenalis - metronidazole - resistance

Giardiasis is a waterborne disease caused by the protozoan Giardia duodenalis (syn. G. lamblia, G. intestinalis) that colonises the small intestine. The infection is worldwide distributed affecting around 280 million people each year most of them children..$^{(1,2)}$ Although the prevalence of giardiasis is high in low income regions, the transmission is also a concern in developed countries, especially as outbreaks. ${ }^{(2)} G$. duodenalis is a zoonotic parasite classified into eight assemblages (A to $\mathrm{H}){ }^{(3)}$ The assemblages $\mathrm{A}$ and $\mathrm{B}$ are classically known to infect humans and dogs. In addition, the assemblage $\mathrm{E}$ infecting humans was also described in $\mathrm{Brazil}^{(4)}$ and Australia. ${ }^{(5)}$ The faecal-oral transmission along with the close interaction with pets increase the risk of $G$. duodenalis infection

There are relatively few therapies available for treatment of giardiasis and 5-nitroimidazoles drugs, mainly metronidazole (MTZ), are first-line treatment. (6,7) MTZ is a pro-drug and need to be metabolised to exert biological activity. In $G$. duodenalis, the proteins pyruvate:ferredoxin oxidoreductase (PFOR) and ferredoxin (Fd) act together to activate MTZ ${ }^{(8)}$ However, two other proteins were also associated with MTZ activation: nitroreductase-1 (NR-1) ${ }^{(9)}$ and thioredoxin reductase (TrxR). ${ }^{(10)}$ MTZ activation generates a nitro anion

\footnotetext{
doi: 10.1590/0074-02760200303

Financial support: IOC/Fiocruz-Brazilian Ministry of Health (internal funds).

$M F$ received funding from Universal/National CNPq, was supported by a

fellowship from CAPES (Brasil sem Miséria), CNPq (PDJ) and is now a

post-doctorate fellow from INOVA Program (Fiocruz); LAPL-O is a

doctorate student supported by CAPES; AMD-C has a research fellowship from CNPq and FAPERJ (CNE).

+ Corresponding author: alda@ioc.fiocruz.br

(D) https://orcid.org/0000-0001-7455-2447

Received 09 June 2020

Accepted 14 October 2020
}

radical that causes oxidative damage to several cellular macromolecules, especially DNA and proteins, leading to the parasite's death. .7,11) $^{(1)}$

The use of 5-nitroimidazoles is well established in clinical practice, the efficacy is high and the estimate cure rate can achieve up to $90 \%$ of the giardiasis treated cases. ${ }^{(6)}$ However, increasing therapeutic failure has been observed in clinical practice. Studies conducted in Spain ${ }^{(12,13)}$ and United Kingdom ${ }^{(14)}$ evaluating travellers point to increasing rates of 5-nitroimidazole refractory Giardia infection. Moreover, in a Cuban cohort of 456 Giardia-microscopically positive individuals, 46\% of them experienced therapeutic failure after the first course of MTZ treatment. ${ }^{(15)}$ Indiscriminate use of antiparasitic drugs together with repeated treatments can increase the chances for rising MTZ-resistant G. duodenalis strains. These strains can spread into the environment, infect susceptible individuals and, consequently, make the treatment of giardiasis more difficult.

The resistance of microorganisms to different classes of drugs is a worldwide concern. Several mechanisms cause resistance to drugs, such as mutation, changes in gene expression and protein abundance, efflux pumps, epigenetic changes and others. Giardia resistance to 5-nitroimidazoles was extensively documented in vitro, but the mechanisms underlying this resistance is still largely unknown. ${ }^{(16-19)}$ Considering the role of PFOR, Fd, NR-1 and TrxR in the MTZ activation, nucleotide alterations in genes that encode these proteins could be associated with 5-nitroimidazoles parasitic resistance. Herein, we evaluated whether in vitro induced MTZ-resistant $G$. duodenalis strain presents mutations in these genes.

Trophozoites of G. duodenalis WB strain [ATCC50803] were grown in TYI-S-33 medium as previously described by Keister ${ }^{(20)}$ supplemented with $10 \%$ of foetal bovine serum (Sigma Chemical Co., Saint Louis, USA) and incubated at $37^{\circ} \mathrm{C}$.

Four groups were defined where G. duodenalis was continuously exposed to different concentrations of MTZ as follow: $5 \mu \mathrm{M}$ (MTZ5); $10 \mu \mathrm{M}$ (MTZ10); $20 \mu \mathrm{M}$ 
(MTZ20); and $80 \mu \mathrm{M}$ (MTZ80). Two groups were used as controls: the group no exposed to MTZ (SMTZ) and the group exposed to $0.05 \%(\mathrm{~V} / \mathrm{V})$ of dimethylsulfoxide (Sigma, USA) (CDMSO), the vehicle in which MTZ was solubilised. After 16 weeks, the $\mathrm{IC}_{50}$ was evaluated for all groups evaluated. ${ }^{(21)}$ Briefly, $5 \times 10^{4}$ parasites from each experimental group were plated in a 96 -well flat-bottom plate, in which a serial dilution of MTZ was carried out. The plates were incubated at $37^{\circ} \mathrm{C}$ for $72 \mathrm{~h}$. Resazurin was added to evaluate the parasite's viability. After $4 \mathrm{~h}$ incubation, the reaction was quantified by fluorimeter $(\lambda$ ex $560 \mathrm{~nm}-\lambda$ em $590 \mathrm{~nm}){ }^{21}$ The tests were performed in triplicate and the $\mathrm{IC}_{50}$ results were analysed using the GrafPad Prism 6.0 software (www.grafpad.com/scientific-software/prism/). The results were expressed as $\mathrm{IC}_{50}$ values and resistance factor, i.e, $\mathrm{IC}_{50}$ fold change in relation to control. The ANOVA test was used for comparative analysis of the $\mathrm{IC}_{50}$ of groups.

The DNA from experimental and control groups were extracted using DNAzol ${ }^{\mathrm{TM}}$ reagent (Qiagen, Hilden, Germany) according to the manufacturer's instructions. The DNA was subjected to PCR to amplify gene fragments. The specific primers for $f d$ (orf. GL50803_9662), $n r-1$ (orf.GL50803 6175) and trxr (orf.GL50803 9827) were designed using the NCBI Pick Primers Tool (https:// www.ncbi.nlm.nih.gov/tools/primer-blast/). The amplified fragments of $n r-1$ (953 base pairs), $f d$ (251 base pairs) and $\operatorname{tr}(1,273$ base pairs) comprised the complete sequence of these proteins. The amplified fragment of pfor (342 base pairs) (orf.GL50803_114608) contains the coding region of the catalytic site of the enzyme. ${ }^{(16)}$ The primers sequences, the concentration of the mix reagents and amplification conditions are described in Table. The effectiveness and efficiency of PCR conditions were assessed by electrophoresis in agarose gel $1 \%$.

The gene fragments obtained in the PCR reaction were purified using the QIAquick ${ }^{\circledR}$ - PCR purification Kit (Qiagen, Germany), according to the manufacturer's instructions. The effectiveness and efficiency of PCR conditions were assessed by electrophoresis in agarose gel $1 \%$. The purified fragments were subjected to sequencing using the BigDye ${ }^{\circledR}$ Terminator v3.1 Cycle Sequencing Kit (Applied Biosystems, Foster City, USA). The precipitation and electrophoresis steps were per- formed at Sequencing Service DNA Sequencing Platform - Fundação Oswaldo Cruz. ${ }^{(22)}$

The electropherograms were analysed and their quality was verified by the Chromas 2.4 program (http:// tecnelysium.com.au/wp/chormas/). The identity percentage was verified by the Basic Local Alignment Search Tool (BLASTn) (https: /blast.ncbi.nlm.nih.gov./ Blast.cgi). The obtained sequences were aligned with the nucleotide sequences of $p f o r, f d, n r-1$ and trxr of $G$. duodenalis in GenBank using the CLUSTAL W algorithm ${ }^{(23)}$ in the Mega 7.0 package (http://www.megasoftware.net) (24) and the consensus obtained by CAP3 Sequence Assembly Program (http://doua.prabi.fb/software/cap3).

To evaluate whether the MTZ exposition decreases the parasite's susceptibility to the drug, $G$. duodenalis trophozoites were continuously exposed to different concentrations of the MTZ during 16 weeks. Afterwards, the parasite's susceptibility to the different MTZ concentrations was evaluated by the rate of trophozoite replication determined by the $\mathrm{IC}_{50}$ values and the resistance factor (Figure). The $\mathrm{IC}_{50}$ values of MTZ5, MTZ10 and MTZ20 groups were significantly higher in comparison to SMTZ and CDMSO groups. This result indicates that G. duodenalis exposition to MTZ induced a reduction of the parasite's susceptibility in a dose dependent manner. $\mathrm{The} \mathrm{IC}_{50}$ values of MTZ80 group could not be determined since the majority of parasites were dead at 16 weeks.

The reduced susceptibility of G. duodenalis trophozoites to MTZ could be caused by alterations in the nucleotide sequence of the genes associated with MTZ activation. Thus, nucleotide sequences of these genes were evaluated. All groups had their DNA amplified and sequenced. The length of the amplified fragments obtained by PCR are the expected for each amplified sequence. No alteration on the nucleotide sequence of $p f o r, f d, n r-1$ and trxr was identified in the parasites from the exposed groups MTZ5, MTZ10, MTZ20, MTZ80 and controls. These results indicate that the reduction of MTZ susceptibility of Giardia trophozoites in vitro is not a consequence of $p f o r, f d, n r-1$ and trxr nucleotide modifications.

Several factors can account for refractory cases of giardiasis after treatment with different drug classes, as inadequate use of drug doses, immunosuppression, reinfection and resistance of the parasite to drugs. In our

TABLE

Oligonucleotide sequence of primers and amplification conditions of the polymerase chain reaction

\begin{tabular}{lcccccccc}
\hline Locus & Sequence(5'-3') & $\begin{array}{c}\mathrm{MgCl}_{2} \\
(\mathrm{mM})\end{array}$ & $\begin{array}{c}\mathrm{Taq} \\
(\mathrm{U})\end{array}$ & $\begin{array}{c}\text { Primer } \\
(\mu \mathrm{M})\end{array}$ & Denaturation & Hybridisation & Extension & $\begin{array}{c}\text { Product } \\
\text { Cycles }\end{array}$ \\
\hline size $(\mathrm{bp})$
\end{tabular}

bp: base pairs. 
A
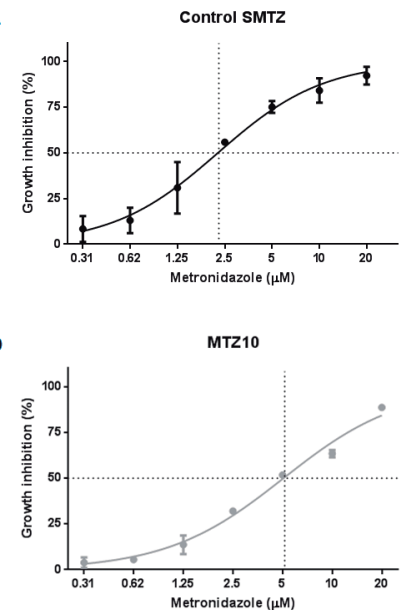

B

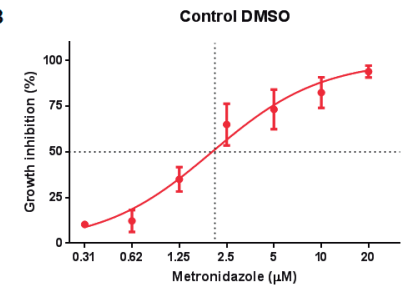

E

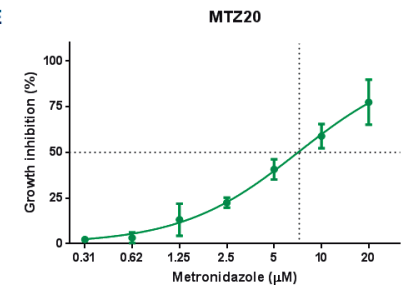

C
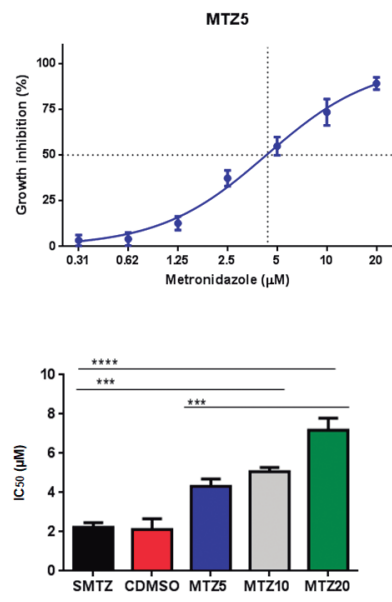

Evaluation of the susceptibility of Giardia duodenalis exposed in vitro to different concentrations of metronidazole (MTZ). The IC ${ }_{50}$ values are indicated on the curves by intersecting MTZ concentration (x-axis) with the $50 \%$ growth inhibition value (y-axis). The graphs are representative of three independent experiments and the results were expressed as mean and standard deviation. (A) No exposed to MTZ (SMTZ) (IC S0 $_{50} .26$ $\pm 0.3 \mu \mathrm{M})$; (B) exposed to dimethylsulfoxide (CDMSO) $\left.\left(\mathrm{IC}_{50}=2.02 \pm 0.3 \mu \mathrm{M}\right) ;(\mathrm{C}) \mathrm{MTZ5}_{(\mathrm{IC}}=4.3 \pm 0.6 \mu \mathrm{M}\right) ;(\mathrm{D}) \mathrm{MTZ10}\left(\mathrm{IC}{ }_{50}=5.07 \pm 0.7\right.$ $\mu \mathrm{M})$; (E) MTZ20 $\left(\mathrm{IC}_{50}=7.09 \pm 0.8 \mu \mathrm{M}\right)$; (F) $\mathrm{IC}_{50}$ values of the groups are compared (ANOVA test). The resistant factor for MTZ5, MTZ10 and MTZ 20 cultures were, respectively, 2.1, 2.5 and 3.5. ***: $\mathrm{p}<0.001 ; * * * *: \mathrm{p}<0.0001$.

study, we induced $G$. duodenalis-resistant trophozoites by in vitro exposition to different concentrations of MTZ and demonstrated that no nucleotide alteration occurred in genes previously associated with Giardia resistance.

The in vitro exposure of $G$. duodenalis to MTZ significantly increased the $\mathrm{IC}_{50}$ values of the groups exposed to this drug compared to controls, similar to the findings of other studies. ${ }^{(16,17,25)}$ Herein, the $\mathrm{IC}_{50}$ fold change values obtained from MTZ exposed parasites ranged from 2.01 to 3.51, as observed to other MTZresistant trophozoites strains. ${ }^{(17)}$ However, our strategy of continuous MTZ exposition differed from other authors who used intermittent exposure of $G$. duodenalis to high concentrations of 5-nitroimidazoles or ultraviolet radiation followed by exposure to this drug. ${ }^{(25)}$ As expected, the results demonstrated that the continuous exposure of $G$. duodenalis to MTZ decreased the parasite's susceptibility to this drug in vitro.

The mechanism responsible for $G$. duodenalis in $v i$ tro resistance to MTZ is still unknown. Since parasite's proteins such as PFOR, Fd, NR-1 and TrxR participate in the activation of MTZ, nucleotide changes in the genes that encode these proteins could prejudice the activation of the drug, leading to a reduction in Giardia susceptibility. This prompted us to evaluate the gene sequences of these proteins in strains of $G$. duodenalis resistant to MTZ in vitro. Unexpected, we did not find any nucleotide change in these analysed genes.

Analysis of transcriptome and proteome of resistant strains of $G$. duodenalis have shown great variability in gene expression and protein abundance of Fd and PFOR. (17,19) However, some studies have detected a decrease in the PFOR enzyme activity ${ }^{(10,26)}$ or the Fd activity in MTZ-resistant $G$. duodenalis strains. ${ }^{(27)} \mathrm{We}$ extended previous studies that showed MTZ-resistant strains of $G$. duodenalis did not present any nucleotide changes in the gene region that encode PFOR catalytic site. ${ }^{(16)}$ Fur- thermore, the expression of pfor mRNA was shown to be higher in the resistant strain of $G$. duodenalis compared to controls. ${ }^{(16)}$ These findings evidence the complexity involving MTZ-G. duodenalis resistance process, which may include another mechanisms. Then, nucleotide changes only in PFOR and Fd are insufficient to elucidate the resistance of $G$. duodenalis to MTZ.

Like Fd and PFOR, gene expression and protein abundance of TrxR showed variable results when MTZresistant $G$. duodenalis strains were evaluated.(17-19) However, NR-1 was downregulated in three G. duodenalis strains resistant to MTZ, ${ }^{(17,19)}$ with evidence of nonsense mutation in NR-1 transcripts in one of strains. (17) This fact demonstrates the importance of evaluating the nucleotide sequence of this gene in different in vitro MTZ-resistant G. duodenalis strains.

Indiscriminate use of antiparasitic drugs, a common practice in health care units, can select resistant strains of several microorganisms. Given the increase in therapeutic failure cases in the treatment of giardiasis with 5-nitroimidazoles, efforts must be made to elucidate which mechanisms may be responsible for the resistance of $G$. duodenalis to MTZ. In the present study, we demonstrated that the exposure of $G$. duodenalis to MTZ decreases the parasite's susceptibility to the drug; however, this decrease could not be attributed to nucleotide changes in the $G$. duodenalis genes linked to MTZ activation. Our results points that different mechanisms as alterations in other metabolic pathways can account for Giardia resistance to MTZ therapy.

\section{ACKNOWLEDGEMENTS}

To Dr Flavia Carneiro, for the critical review, to Prof Marlene Benchimol, who kindly provide us the cells of the G. duodenalis strain WB ATCC 50803, to the Trypanosomatid Biochemistry Laboratory-Fiocruz, for providing the MTZ used in this study. The samples were analysed by the Sequencing Service DNA Sequencing Platform-Fiocruz. 


\section{AUTHOR'S CONTRIBUTION}

LAPL-O and MF were responsible for the study design, experiments and obtaining results, analysis of the results and writing the manuscript. AMC was responsible for funding, review of the results and writing and final approval of the paper. The authors declare no conflicts of interest.

\section{REFERENCES}

1. Lane S, Lloyd D. Current trends in research into the waterborne parasite Giardia. Crit Rev Microbiol. 2002;28(2):123-47.

2. Lalle M. Giardiasis in the post genomic era: treatment, drug resistance and novel therapeutic perspectives. Infec Disord Drug Targets. 2010;10(4):283-94.

3. Feng Y, Xião L. Zoonotic potential and molecular epidemiology of Giardia species and giardiasis. Clin Microbiol Rev. 2011;24(1):110-40.

4. Fantinatti M, Bello AR, Fernandes O, da-Cruz AM. Identification of Giardia lamblia assemblage E in humans points to a new anthropozoonotic cycle. J Infect Dis. 2016;214(8):1256-9.

5. Zahedi A, Field D, Ryan U. Molecular typing of Giardia duodenalis in humans in Queensland - First report of assemblage E. Parasitology. 2017;144(9):1154-61.

6. Tejman-Yarden N, Eckman L. New approaches to the treatment of giardiasis. Curr Opin Infect Dis. 2011;24(5):451-6.

7. Vivancos V, González-Alvareza I, Bermejoand M, Gonzalez-Alvareza M. Giardiasis: characteristics, pathogenesis and new insights about treatment. Curr Top Med Chem. 2018;18(15):1287-303.

8. Edwards DI. Nitroimidazole drugs-action and resistance mechanisms I. Mechanisms of action. J Antimicrob Chemother. 1993;31(1):9-20.

9. Nillius D, Müller J, Müller N. Nitroreductase (G1NR1) increases susceptibility of Giardia lamblia and Escherichia coli to nitro drugs. J Antimicrob Chemother. 2011;66(5):1029-35.

10. Leitsch D, Burgess AG, Dunn LA, Krauer KG, Tan K, Duchêne $\mathrm{M}$, et al. Pyruvate:ferredoxin oxidoreductase and thioredoxin reductase are involved in 5-nitroimidazole activation while flavin metabolism is linked to 5-nitroimidazole resistance in Giardia lamblia. J Antimicrob Chemother. 2011;66(8):1756-65.

11. Freeman CD, Klutman NE, Lamp KC. Metronidazole. A therapeutic review and update. Drugs. 1997;54(5):679-708.

12. Muñoz Gutiérrez J, Aldasoro E, Requena A, Comin AM, Pinazo MJ, Bardají A, et al. Refractory giardiasis in Spanish travellers. Travel Med Infect Dis. 2013;11(2):126-9.

13. Requena-Méndez A, Goñi P, Lóbez S, Oliveira I, Aldasoro E, Valls ME, et al. A family cluster of giardiasis with variable treatment responses: refractory giardiasis in a family after a trip to India. Clin Microbiol Infect. 2014;20(2):135-8.
14. Nabarro LE, Lever RA, Armstrong M, Chiodini PL. Increased incidence of nitroimidazole-refractory giardiasis at the Hospital for Tropical Diseases, London: 2008-2013. Clin Microbiol Infect. 2015;21(8):791-6.

15. Cañete R, Noda AL, Rodríguez M, Brito K, Herrera E, Kofoed PE, et al. 5-Nitroimidazole refractory giardiasis is common in Matanzas, Cuba and effectively treated by secnidazole plus high-dose mebendazole or quinacrine: a prospective observational cohort study. Clin Microbiol Infect. 2019;S1198-743X(19)30674-3.

16. Argüello-García R, Cruz-Soto M, Romero-Montoya L, OrgetaPierres G. In vitro resistance to 5-nitroimidazoles and bezimidazoles in Giardia duodenalis: variability and variation in gene expression. Infect Genet Evol. 2009;9(6):1057-64.

17. Ansell BRE, Baker L, Emery SJ, McConville MJ, Svärd SG, Gasser AR, et al. Transcriptomics indicates active and passive metronidazole resistance mechanisms in three seminal Giardia lines. Front Microbiol. 2017;8:398.

18. Müller J, Braga S, Heller M, Müller N. Resistance formation to nitro drugs in Giardia lamblia: no common markers identified by comparative proteomics. Int J Parasitol Drugs Drug Resist. 2019;9:112-9.

19. Emery SJ, Baker L, Ansell BRE, Mirzaei M, Haynes PA, McConville MJ, et al. Differential protein expression and post-translational modifications in metronidazole-resistant Giardia duodenalis. Gigascience. 2018;7(4):giy024.

20. Keister DB. Axenic culture of Giardia lamblia in TYI-S-33 medium supplemented with bile. Trans R Soc Trop Med Hyg. 1983;77(4):487-8.

21. Bénéré E, da Luz RA, Vermeersch M, Cos P, Maes L. A new quantitative in vitro microculture method for Giardia duodenalis trophozoites. J Microbiol Methods. 2007;71(2):101-6.

22. Otto TD, Catanho M, Degrave W, Miranda AB. The PDTIS bioinformatics platform: from sequence to function. RECIIS- Elet $J$ Commun Inf Innov Health. 2007;1(2):286-94.

23. Thompson JD, Higgins DG, GibsonTJ. CLUSTAL W: improving the sensitivity of progressive multiple sequence alignment through sequence weighting, position-specific gap penalties and weight matrix choice. Nucleic Acids Res. 1994;22(22):4673-80.

24. Kumar S, Stecher G, Tamura K. MEGA7: Molecular Evolutionary Genetics Analysis version 7.0 for bigger datasets. Mol Biol Evol. 2016;33(7):1870-4.

25. Townson SM, Laqua H, Upcroft P, Boreham PFL, Upcroft JA. Induction of metrodinazole and furazolidone resistence in Giardia. Trans R Soc Trop Med Hyg. 1992;86(5):521-2.

26. Townson SM, Upcroft JA, Upcroft P. Characterisation and purification of pyruvate:ferredoxin oxidoreductase from Giardia duodenalis. Mol Biochem Parasitol. 1996;79(2):183-93.

27. Liu SM, Brown DM, O’Donoghue P, Upcroft P, Upcroft JA. Ferredoxin involvement in metronidazole resistance of Giardia duodenalis. Mol Biochem Parasitol. 2000;108(1):137-40. 